\title{
Transient Pupil Response Is Modulated by Contrast-Based Saliency
}

\author{
Chin-An Wang, ${ }^{1}$ Susan E. Boehnke, ${ }^{1}$ Laurent Itti, ${ }^{2}$ and Douglas P. Munoz ${ }^{1}$ \\ ${ }^{1}$ Centre for Neuroscience Studies, Queen's University, Kingston, Ontario K7L 3N6, Canada, and ${ }^{2}$ Department of Computer Science, University of Southern \\ California, Los Angeles, California 90089-0781
}

\begin{abstract}
The sudden appearance of a novel stimulus in the environment initiates a series of orienting responses that include coordinated shifts of gaze and attention, and also transient changes in pupil size. Although numerous studies have identified a significant effect of stimulus saliency on shifts of gaze and attention, saliency effects on pupil size are less understood. To examine salience-evoked pupil responses, we presented visual, auditory, or audiovisual stimuli while monkeys fixated a central visual spot. Transient pupil dilation was elicited after visual stimulus presentation regardless of target luminance relative to background, and auditory stimuli also evoked similar pupil responses. Importantly, the evoked pupil response was modulated by contrast-based saliency, with faster and larger pupil responses following the presentation of more salient stimuli. The initial transient component of pupil dilation was qualitatively similar to that evoked by weak microstimulation of the midbrain superior colliculus. The pupil responses elicited by audiovisual stimuli were well predicted by a linear summation of each modality response. Together, the results suggest that the transient pupil response, as one component of orienting, is modulated by contrast-based saliency, and the superior colliculus is likely involved in its coordination.
\end{abstract}

Key words: microstimulation; orienting; pupil response; saliency; stimulus contrast; superior colliculus

\section{Introduction}

Sensory systems must efficiently select crucial elements from the excessive information available in the environment (Desimone and Duncan, 1995). This selection process is greatly influenced by a bottom-up, saliency-based mechanism (Itti and Koch, 2001), in which the saliency or conspicuity of objects (or locations) in the environment is encoded, and the most salient item triggers an orienting response to allocate neuronal resources toward that item for computationally intensive processing.

Saccadic eye movements and attention shifts, as components of the orienting response, are evoked by salient stimulus presentation and are modulated by various aspects of stimulus saliency (Carrasco, 2011; Kowler, 2011). Computational models have been remarkably successful at demonstrating these saliency effects (Koch and Ullman, 1985; Itti and Koch, 2000; Parkhurst et al., 2002; Berg et al., 2009; Borji et al., 2013). Although the orienting response also includes transient pupil responses (Sokolov, 1963b; Boehnke and Munoz, 2008), modulation of pupil dynam-

\footnotetext{
Received Aug. 19, 2013; revised Nov. 10, 2013; accepted Nov. 19, 2013.

Author contributions: C.-A.W., S.E.B., L.I., and D.P.M. designed research; C.-A.W., S.E.B., and L.I. performed research; C.-A.W. contributed unpublished reagents/analytic tools; C.-A.W. and L.I. analyzed data; C.-A.W., S.E.B., L.I., and D.P.M. wrote the paper.

This work was surported by Canadian Institutes of Health Research Grant MOP-77734 and the Defense Advanced Research Projects Agency Neovision2 Program Grant, and the National Science Foundation (Grant nos. CCF-1317433 and (MMI-1235539). C.-A.W. was supported by The Natural Sciences and Engineering Research Council of Canada (NSERC) Collaborative Research and Training Experience (CREATE) Program and D.P.M. was supported by the Canada Research Chair Program. We thank Ann Lablans, Donald Brien, Sean Hickman, and Mike Lewis for outstanding technical assistance, as well as members of the Munoz lab for comments on an earlier version.

Correspondence should be addressed to Dr Chin-An Wang, Centre for Neuroscience Studies, Queen's University, Room 245, Botterell Hall, 18 Stuart Street, Kingston, 0N K7L 3N6, Canada. E-mail: josh.wang@queensu.ca.

DOI:10.1523/JNEUROSCI.3550-13.2014

Copyright $\odot 2014$ the authors $\quad 0270-6474 / 14 / 340408-10 \$ 15.00 / 0$
}

ics by saliency remains poorly understood. Nevertheless, pupil size has been widely used to assess cognitive processing (Beatty, 1982; Nassar et al., 2012; Wierda et al., 2012; Eldar et al., 2013).

The superior colliculus (SC) is a phylogenetically well preserved subcortical structure (Ingle, 1973; Hall and Moschovakis, 2003) known for its central role in saccadic eye movement initiation and attentional shifts (Schiller et al., 1987; McPeek and Keller, 2004; Krauzlis et al., 2013), and in multisensory processing (Stein and Meredith, 1993). The SC is hypothesized to encode stimuli based upon saliency to coordinate all components of the orienting response (Sparks, 1986; Fecteau and Munoz, 2006; Knudsen, 2007; Boehnke and Munoz, 2008; Mysore and Knudsen, 2013). Recently, it was shown that SC microstimulation evokes pupil dilation, extending the role of the SC to pupil dynamics (Netser et al., 2010; Wang et al., 2012), although the function of this transient pupil response is still unclear. If the SC encodes stimulus saliency to coordinate an orienting response that includes transient pupil responses, then the pupil should respond to both visual and auditory stimuli, and the saliency effects on pupil dilation should be comparable to such effects on sensory-evoked responses in the SC.

Here we examine pupil responses elicited in behaving rhesus monkeys following the presentation of visual, auditory, or audiovisual stimuli. Transient pupil dilation was consistently evoked by a visual stimulus defined by a luminance increment or decrement relative to background. The pupil responded similarly to visual and auditory stimuli, and was modulated by contrastbased saliency, with faster and larger pupil responses observed for higher contrast stimuli. Moreover, the evoked transient pupil dilation was qualitatively similar to that elicited by SC stimulation (Wang et al., 2012). Computationally, pupil responses to 
audiovisual stimuli are well approximated by the sum of the responses evoked by the stimulus components. Overall, our results demonstrate the effects of contrast-based saliency modulation on transient pupil responses, and suggest that the SC may coordinate such behavior.

\section{Materials and Methods}

Animal preparation and equipment. All protocols used in this study were approved by Queen's University Animal Care Committee in accordance with the Canadian Council on Animal Care policies on the use of laboratory animals. Experiments were performed on four male rhesus monkeys (Macaca mulatta; 10-12 kg). Specifically, monkeys U and D were trained to perform the visual and auditory stimulus experiments. The microstimulation experiment was performed on monkeys $\mathrm{Q}$ and $\mathrm{Y}$, and these data were published previously (Wang et al., 2012). The methods of surgical procedures and data collection have been described in detail previously (Marino et al., 2008). Eye position was measured by the scleral search coil technique (Robinson, 1963), and horizontal and vertical eye positions were digitized at $1000 \mathrm{~Hz}$. Pupil diameter was measured using a video-based eye tracker (Eyelink-II, SR Research) at a rate of $500 \mathrm{~Hz}$.

To calibrate actual pupil size derived from output pupil diameter values recorded from the Eyelink II, we used the following method (Steiner and Barry, 2011; Wang et al., 2012). We made a number of different-sized false pupils ( 6 pupils from 1 to $6 \mathrm{~mm}$ in diameter) and placed them at the exact same position as the monkey's pupil position during data recording. Eyelink pupil values from false pupils were used to transform Eyelink pupil values recorded from real monkeys to actual pupil diameter, and pupil size resolution was $\sim 0.01 \mathrm{~mm}$. Critically, pupil-size data can be distorted by eye movements because the size of the pupil depends on the subject's gaze angle in a video-based eye tracker. In addition, saccade generation could confound our test of the role of stimulus saliency on the evoked pupil responses, because any observed differences in pupil response between different salient conditions could be triggered by saccadic eye movement itself, rather than stimulus saliency per se. To maintain an accurate measure of pupil size before, during, and after stimulation, and to avoid an influence of the saccadic eye movements, monkeys were required to maintain visual fixation on a point at the center of the screen throughout the trial except for the trials that required saccadic eye movements.

Stimulus presentation and data acquisition were controlled by a UNIX-based real-time data control system (REX; Hays et al., 1982). Eye position and pupil diameter were recorded in a multichannel data acquisition system (Plexon). Stimuli were presented on a CRT monitor at a screen resolution of $1024 \times 768$ pixels $(75 \mathrm{~Hz}$ noninterlaced), subtending a viewing angle of $54^{\circ} \times 44^{\circ}$. Luminance measures for visual stimuli were determined using a luminance meter (CS100, Konica Minolta), and sound pressure levels (SPLs) for auditory stimuli were determined using a sound level meter (type 2239, Bruel and Kjaer).

Visual stimulus task. Two monkeys ( $\mathrm{U}$ and $\mathrm{D}$ ) were trained to perform a visual stimulus task (Fig. $1 A, B$ ), similar to previous descriptions (Wang et al., 2012). Each monkey was required to maintain gaze within $1.5^{\circ}$ of a central fixation point ( $\mathrm{FP} ; 0.5^{\circ}$ diameter; $30 \mathrm{~cd} / \mathrm{m}^{2}$ ) for reward. After the monkey maintained a central fixation for $1.4-1.8 \mathrm{~s}$, a peripheral visual stimulus $\left(0.5^{\circ}\right.$ diameter $)$ was presented for $100 \mathrm{~ms}$ to the left or right of the FP on a subset of trials $(33 \%)$ and the monkey was required to maintain steady fixation for an additional $1.4-1.6 \mathrm{~s}$ to obtain reward. The repetition of a same stimulus repeatedly can greatly attenuate the stimulus-evoked pupil responses via habituation (Netser et al., 2010; Steiner and Barry, 2011). We therefore included a no stimulus control condition (Ctrl) on a subset of trials $(33 \%)$ to reduce the effects. In addition, to prevent the monkey from strategically ignoring the peripheral visual stimulus, on another proportion of trials (33\%, data not shown), the FP was removed coincident with visual stimulus appearance, and the monkey was required to generate a saccade toward the stimulus within $500 \mathrm{~ms}$ for reward (Fig. 1A,B; data not shown). These saccade responses were not included in the pupil analysis.

Pupil size is sensitive to level of illumination, with a constriction following increases in illumination and dilation following decreases in illu-

\section{A Light background}

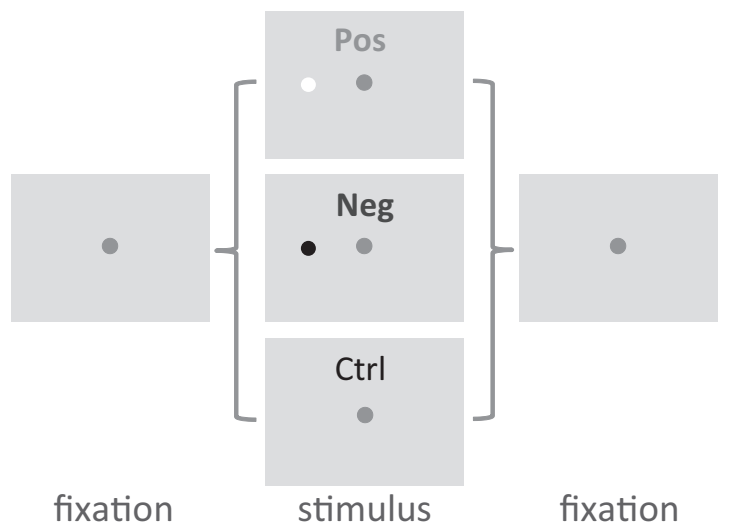

B Dark background

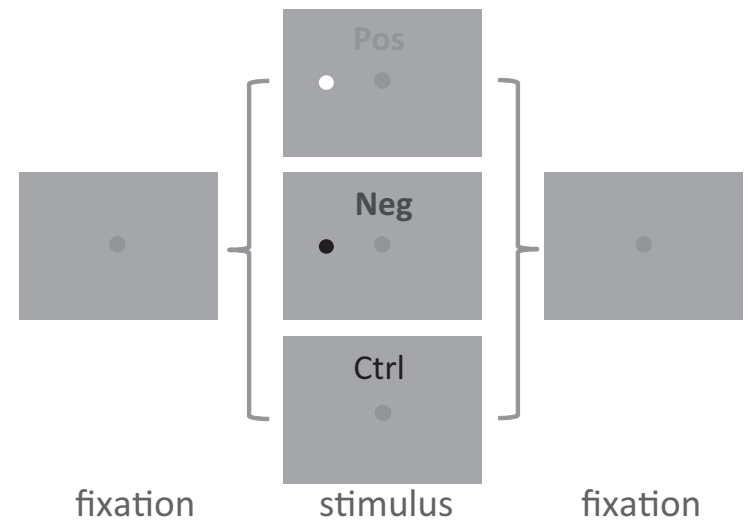

C Pupil response measurement

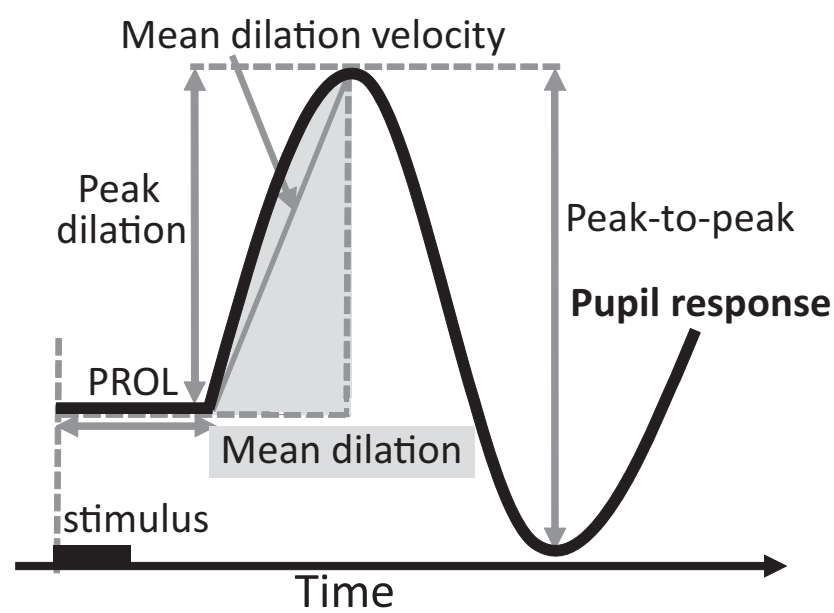

Figure 1. $\quad \boldsymbol{A}, \boldsymbol{B}$, Each trial started with a central fixation point on a dark $(\boldsymbol{A})$ or light $(\boldsymbol{B})$ background. After a random delay there was a brief presentation $(100 \mathrm{~ms})$ of either a positive or negative contrast visual stimulus (Pos/Neg) or no stimulus presented (Ctrl). C, Measurements of the evoked pupil response. PROL, Pupil response onset latency.

mination (Loewenfeld, 1999). To confirm that transient stimulusevoked pupil responses could be induced independent of background illumination, two different values of background luminance were used (35 or $25 \mathrm{~cd} / \mathrm{m}^{2}$; Fig. $1 A$, referred to as light or $B$, dark background). Additionally, to rule out the possibility that pupil responses induced by visual stimuli were driven by a sudden change of overall illumination due to a short presentation of luminant stimuli, we compared pupil responses evoked by a stimulus defined by a luminance increment or decrement 
(Fig. $1 A, B$ ) relative to background (positive/negative contrast: 58 or 16 $\mathrm{cd} / \mathrm{m}^{2}$ on a light background, and 40 or $12 \mathrm{~cd} / \mathrm{m}^{2}$ on a dark background). If pupil responses elicited by salient stimuli were driven by illumination changes, the pupil responses induced by a positive or negative contrast stimulus should be qualitatively different because one decreases but another increases overall illumination. All conditions were randomly interleaved. Data collected across days/sessions were collapsed for analysis.

We selected $10-15^{\circ}$ eccentricity on the horizontal axis to present the visual stimulus because our tasks required the monkey either to make a saccade, or to maintain a fixation following a target presentation, and monkeys typically have difficulty suppressing eye movements to targets at $<5^{\circ}$ eccentricity without extensive overtraining. Additionally, we wanted to mimic the conditions used for our SC microstimulation experiment, which had an average stimulation vector of $\sim 12.5^{\circ}$ (Wang et al., 2012). To confirm that the effects of evoked pupil responses were consistent across different stimulus eccentricities, we conducted a separate experiment in monkey $\mathrm{U}$ using two stimulus eccentricities $\left(10^{\circ}\right.$ and $20^{\circ}$ ). Qualitatively similar pupil responses were induced regardless of eccentricity (Fig. 2G). Moreover, to examine effects of stimulus hemifield on the left and right pupils, we recorded both pupils simultaneously. Presentation of salient stimuli produced consensual pupil responses regardless of stimulus visual field (Fig. $2 \mathrm{H}$ ).

Visual intensity task. Two monkeys ( $\mathrm{U}$ and $\mathrm{D})$ were trained to perform a task similar to the visual stimulus task (Fig. $1 A, B$ ), except the background luminance was held constant $\left(20\right.$ or $\left.24 \mathrm{~cd} / \mathrm{m}^{2}\right)$ and the visual target intensity was manipulated to examine the effects of saliency modulation on evoked transient pupil responses (fixation trials 33\%) and saccade reaction times (saccade trials 33\%). Visual contrast was manipulated because it is one of the most primitive components of saliency (Itti and Koch, 2001), and has been implemented widely in a number of computational models (Borji et al., 2013). There was also a subset of trials with no stimulus presented as the control condition (33\%).

The pupil response was elicited regardless of stimulus luminance relative to background, therefore luminance decrement stimuli were arbitrarily used. The stimulus was either near black (high-contrast, 0.1-4 $\mathrm{cd} / \mathrm{m}^{2}$ ) or gray (low-contrast, $9-12 \mathrm{~cd} / \mathrm{m}^{2}$ ) against a light gray background. All conditions were randomly interleaved and data collected across sessions were collapsed for further analysis.

Auditory intensity task. Monkeys $\mathrm{U}$ and $\mathrm{D}$ were also trained to perform an auditory intensity task. This task was similar to the visual intensity task, except a salient auditory tone was presented. Although tones are notoriously hard to localize, they are best localized at $0^{\circ}$ azimuth and for frequencies less than $\sim 1200 \mathrm{~Hz}$ for which the interaural time difference can be exploited (Middlebrooks and Green, 1991), even though frontback confusions likely occurred. Moreover, tonal signals have the advantage of high saliency against the white noise background of the lab environment, and have been used previously (Bala and Takahashi, 2000). Although the monkey fixated the central FP $\left(0.5^{\circ}\right.$ diameter; $20-23 \mathrm{~cd} /$ $\mathrm{m}^{2}$ ) for reward, on half the trials an auditory stimulus (100 ms pure tone at $1000 \mathrm{~Hz}$ generated by the Matlab software, MathWorks) was presented at $0^{\circ}$ azimuth from a speaker located above the monitor. The intensity of the auditory stimulus (saliency) was also manipulated ( 89 and $82 \mathrm{~dB}$ SPL, background noise was $50 \mathrm{~dB}$ ). All conditions were randomly interleaved and data collected across sessions were collapsed for analysis. The onset and offset of the tone was gated electronically using REX to maintain millisecond-accurate timing.

Audiovisual task. Monkey $\mathrm{U}$ was trained to perform an audiovisual task similar to the visual intensity task, except an auditory stimulus was presented simultaneously with the visual stimulus (V0A), $100 \mathrm{~ms}$ after the visual stimulus (V100A), or $100 \mathrm{~ms}$ before the visual stimulus (A100V). In addition, there were two unisensory conditions (visual or auditory alone). All other procedures were the same as the visual intensity task. Briefly, the monkey fixated the central FP $\left(0.5^{\circ}\right.$ diameter; 20 $\mathrm{cd} / \mathrm{m}^{2}$ ) to obtain a reward. On $33 \%$ of trials, no stimulus was presented (Ctrl), on another $33 \%$ of trials, visual, auditory, or audiovisual stimuli were presented, and on the remaining $33 \%$ of trials, the visual stimulus was presented coincident with the FP disappearance, and the monkey was required to generate a saccade toward the stimulus for reward. In all cases, the auditory stimulus ( $1000 \mathrm{~Hz}$ tone) was presented for $100 \mathrm{~ms}$ at
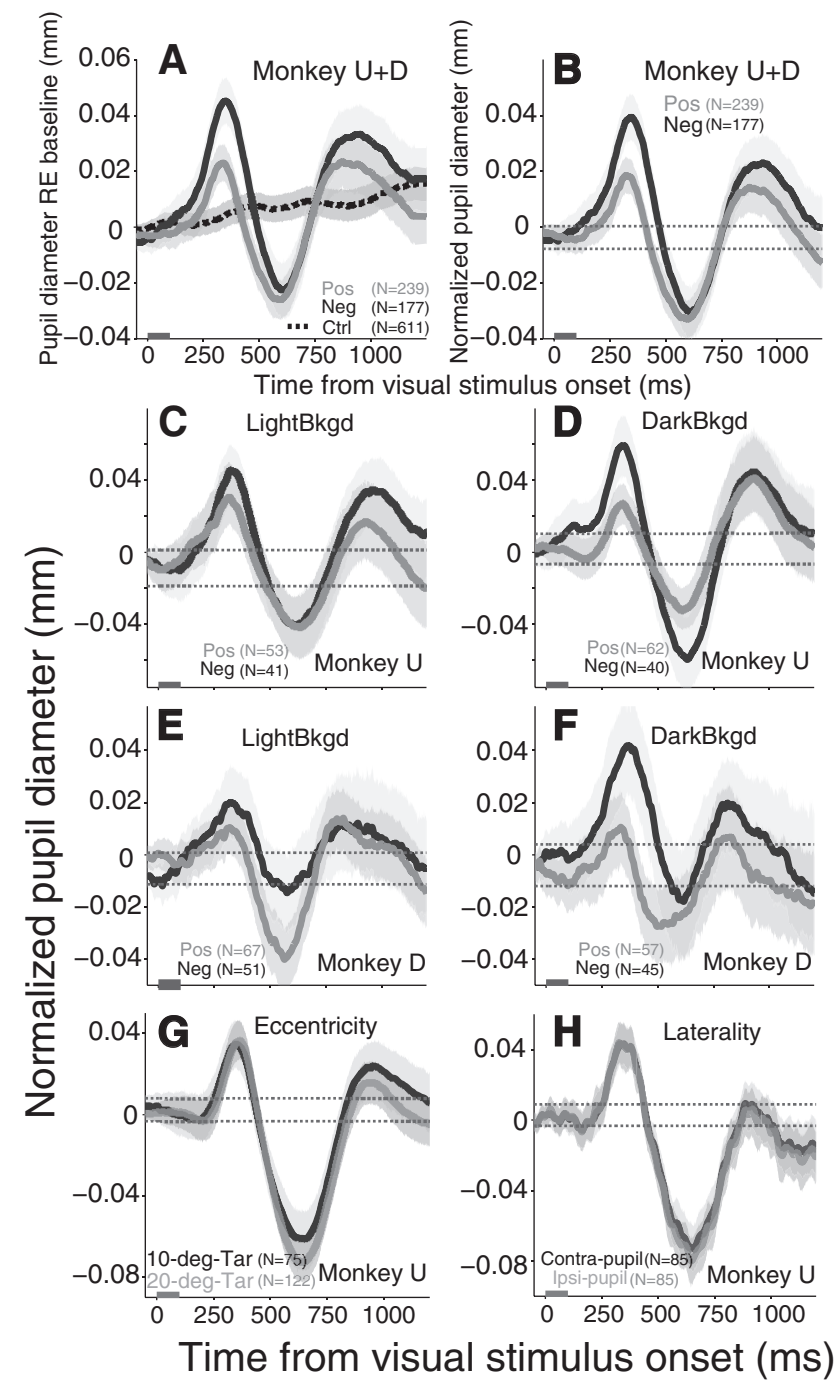

Figure 2. Transient pupil response evoked by brighter or dimmer visual stimuli relative to background. $A$, Pupil response relative to baseline after the presentation of salient visual stimulus. $B$, Pupil response was normalized (stimulus minus no-stimulus conditions). $\boldsymbol{C}, \boldsymbol{E}$, Normalized pupil response after salient visual stimulus on lighter background in monkey $U$ and $D$, respectively. $\boldsymbol{D}, \boldsymbol{F}$, Normalized pupil response after salient visual stimulus on darker background in monkey $\mathrm{U}$ and $\mathrm{D}$, respectively. $\mathbf{G}$, Effects of the eccentricity of visual stimulus on the evoked pupil response. $\boldsymbol{H}$, Summary of pupil effects on the contralateral or ipsilateral pupil of the stimulus visual field. The gray bar on $x$-axis indicates the time line of stimulus presentation. The shaded regions surrounding evoked pupillary response represent $\pm 95 \%$ confidence range from the bootstrap analyses. $\boldsymbol{A}-\boldsymbol{F}$, The brighter and dimmer lines indicate the positive and negative contrast stimulus (Pos/ $/ \mathrm{Neg}$ ) trials, respectively. $A$, Black dotted line indicates trials with no stimulus presentation. $\boldsymbol{B}-\mathbf{G}$, Dotted lines indicate the baseline $95 \% \mathrm{Cl}$, any observed value above or below the dotted lines region indicate the value is statistically significant. $\mathbf{G}$ Black and gray lines indicate $10^{\circ}$ and $20^{\circ}$ stimulus trials, respectively. $\mathbf{H}$, Black and gray lines indicate the contralateral and ipsilateral pupil of the stimulus visual field trials, respectively. $N=$ number of trials. Ctrl, Control condition (no stimulus).

zero azimuth from a speaker located above the monitor at a level of $89 \mathrm{~dB}$ SPL (background noise was $50 \mathrm{~dB}$ ). The visual stimulus was presented for $100 \mathrm{~ms}$ to the right or left of central FP at $10^{\circ}$ eccentricity on the horizontal axis $\left(9 \mathrm{~cd} / \mathrm{m}^{2}, 0.5^{\circ}\right.$ diameter $)$, and the background luminance was held constant $\left(24 \mathrm{~cd} / \mathrm{m}^{2}\right)$. All conditions were randomly interleaved and data collected across sessions were collapsed for analysis.

Note that we did not manipulate the location of the tonal auditory stimulus relative to the visual stimulus; it was always at $0^{\circ}$ azimuth. Therefore, the task made no attempt to examine multisensory integration directly (Stein and Meredith, 1993), but simply investigated the pupil response evoked by audiovisual stimuli from a purely mechanistic perspective. 

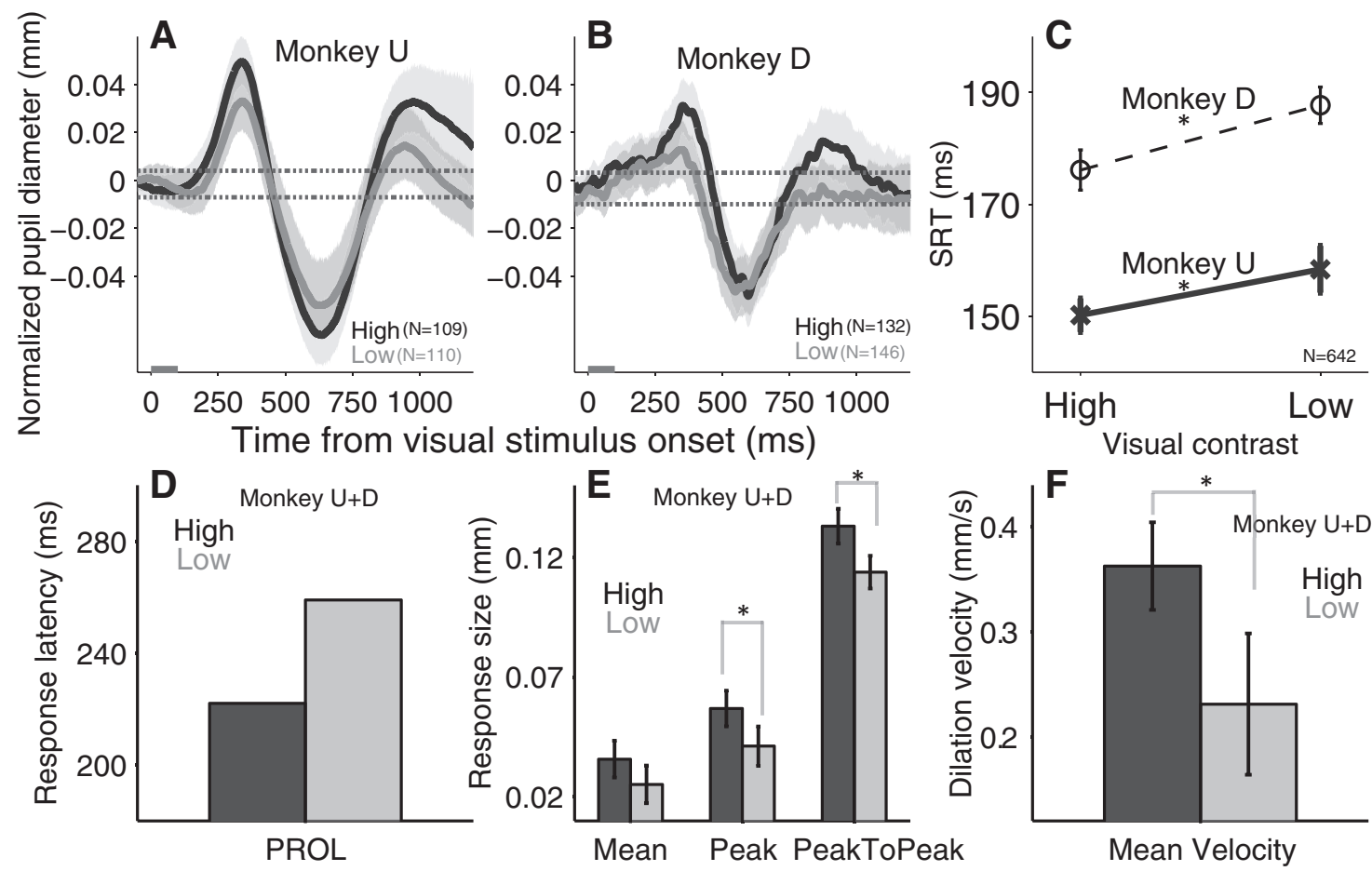

Figure 3. Effect of visual contrast-based saliency modulation on transiently evoked pupil responses. $A, B$, Normalized pupil dynamics after two levels of visual stimulus presentation (highcontrast and low-contrast) in monkey $U$ and $D$, respectively. $C$, Saccade reaction times between the high and the low contrast conditions on different monkeys. $D-F$, Modulation of stimulus contrast on the PROL (D); the mean size of pupil dilation, the peak size of pupil dilation, and peak-to-peak size of pupil response $(\boldsymbol{E})$; and the mean velocity of pupil dilation $(\boldsymbol{F})$. $\boldsymbol{A}, \boldsymbol{B}$, Black and gray lines indicate the high and low contrast stimulus trials, respectively. Shaded regions surrounding evoked pupillary response traces represent $\pm 95 \% \mathrm{Cl}$. Gray bar on $x$-axis indicates the time line of stimulus presentation and the dotted lines indicate the baseline $\pm 95 \%$ confidence range. $\boldsymbol{C}, \boldsymbol{E}, \boldsymbol{F}$, Error bars represent $\pm 95 \% \mathrm{Cl}$. D- $\boldsymbol{F}$, Dark and light bars indicate the high- and low-stimulus contrast condition, respectively. *Differences are statistically significant (not overlap in the compared Cls). SRT, Saccade reaction times; High, high-contrast stimulus; Low, low-contrast stimulus; $N=$ number of trials.

SC microstimulation task. Aspects of the microstimulation data reported herein including detailed methods were published previously using different analysis techniques (Wang et al., 2012). Briefly, two monkeys (Q and Y) were trained to perform a simple fixation task, except instead of presenting a sensory stimulus, a train of microstimulation pulses $(100 \mathrm{~ms}, 300 \mathrm{~Hz}$; range, $5-50 \mu \mathrm{A}$ ) was delivered to the intermediate layers of the SC (SCi) on a subset of trials (50\%). To get an accurate estimation of pupil change linked to microstimulation, we used current values that were $50-70 \%$ of the threshold current to evoke saccades, so that no eye movements were triggered. Note that the latency of microstimulation evoked pupil dilation would be shorter if suprathreshold microstimulation was used. On another $50 \%$ of trials, there was no microstimulation and the monkey was required to maintain central fixation for reward. Luminance levels for FP and background were identical to the visual stimulus task, and all conditions were randomly interleaved. Microstimulation was delivered to 28 sites in the SCi (18 and 10 in monkeys $\mathrm{Q}$ and $\mathrm{Y}$, respectively).

Data analysis. The initial transient component of the evoked pupil response was of primary interest here (Fig. 1C). Therefore, the analyses focused on the initial transient pupil response. Trials with blinks or an eye position deviation of $>1.5^{\circ}$ from the central FP during the required period of fixation were excluded from analysis. Because the pupil response was confirmed to be consensual, only pupil diameter of the right eye was recorded for data analysis. The same procedure was used to quantify pupil responses previously (Wang et al., 2012). For each trial, original pupil diameter values were subtracted from the baseline pupil diameter value determined by averaging pupil size from $250 \mathrm{~ms}$ before to the onset of stimulation (visual/auditory/electrical; Bala and Takahashi, 2000; Moresi et al., 2008). Because pupil size increased slightly even when there was no stimulus presented (Fig. $2 A$, dotted line), to simplify data presentation and quantification, we normalized pupil diameter values by contrasting the sensory/electrical stimulation (Fig. $2 A$, solid line) versus no-stimulation (Fig. 2A, dotted line) conditions directly. Specifically, pupil values from each stimulation trial were contrasted to the average pupil value from all no-stimulation trials (Fig. 2A,B).

In contrast to our previous study (Wang et al., 2012), we used a bootstrap method to inform the statistical significance of the comparison by performing a random sampling of pupil values derived from each recording trial with 1500 repetitions. This resulted in a normally distributed cluster of points centered on the mean of selected pupil values (clusters not shown, normal distribution was verified by the Kolmogorov-Smirnov test). The 95\% confidence interval $(\mathrm{CI})$ derived from the bootstrap analysis was depicted in shading (Fig. 3A) or in error bar (Fig. 3B), and the planned comparison was considered to be statistically significant when two compared CIs did not overlap. The baseline CI (Fig. $2 B-H$, dotted lines) was defined by the onset pupil value calculated by averaging pupil values from higher or lower $95 \% \mathrm{CI}$ range from $25 \mathrm{~ms}$ before to $25 \mathrm{~ms}$ after stimulation ( -25 to $\sim 25 \mathrm{~ms}$ ), and a pupil value at any given time exceeded the baseline CI range was considered to be statistically significant.

Figure $1 C$ shows a number of measurements we extracted to capture dynamics of evoked transient pupil responses (Beatty and LuceroWagoner, 2000). The pupil response onset latency (PROL) was computed using the average of all trials in a given condition rather than trial-by-trial due to a low signal-to-noise ratio. PROL was defined as the earliest point at which average pupil magnitude exceeded the baseline CI range. The remaining pupil measurements were computed on a trial-bytrial basis. We also characterized several size-related pupil measurements. The mean dilation was defined as the average of the pupil size from PROL to the time where the pupil reached to the highest value within $400 \mathrm{~ms}$ (arbitrarily selected from visual inspection) after the stimulation onset (visual/auditory/electrical). The peak dilation was defined as the maximum value observed within $400 \mathrm{~ms}$ after the stimulation onset. The peak-to-peak pupil response was defined as the peak dilation minus the pupil size at the time where the pupil reached to the lowest value within $350 \mathrm{~ms}$ after the peak dilation (arbitrarily selected from 

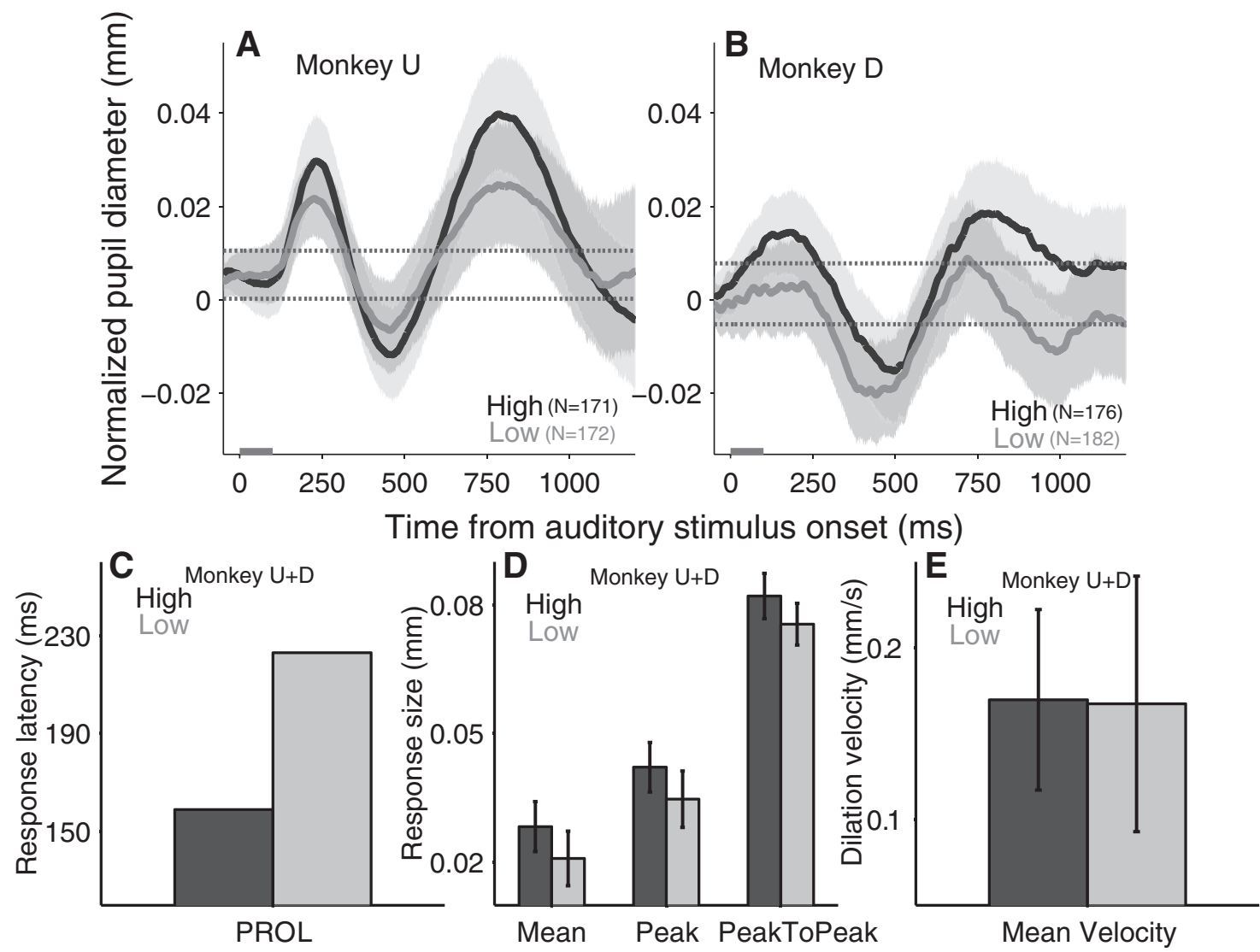

Figure 4. Effect of auditory contrast-based saliency modulation on transiently evoked pupil responses. $A, B$, Pupil response evoked by auditory stimuli (high-and low-intensity) in monkey $U$ and $D$, respectively. $\boldsymbol{C}-\boldsymbol{E}$, Modulation of auditory stimulus intensity on the PROL (C); the mean size of pupil dilation, the peak size of pupil dilation, and peak-to-peak size of pupil response (D); and the mean velocity of pupil dilation $(\boldsymbol{E}) . \boldsymbol{A}, \boldsymbol{B}$, Black and gray lines indicate the high- and low-contrast stimulus trials, respectively. Shaded regions surrounding evoked pupillary response traces represent $\pm 95 \% \mathrm{Cl}$. Gray bar on $x$-axis indicates the time line of stimulus presentation and the dotted lines indicate the baseline $\pm 95 \%$ confidence range. $\boldsymbol{D}, \boldsymbol{E}$, Error bars represent $\pm 95 \% \mathrm{Cl}$. C $\boldsymbol{E}$, Dark and light bars indicate the high and low auditory-stimulus intensity condition, respectively. High, High-auditory intensity stimulus; Low, low-auditory intensity stimulus; $N=$ number of trials.

visual inspection). The mean dilation velocity was computed using the same time window for the mean pupil dilation (positive and negative values indicate the dilating and constricting process, respectively).

Computational modeling. We considered a simple linear model to analyze pupil responses to audiovisual stimuli. This "additive" model simply summed the responses obtained separately from each sensory modality, after proper temporal alignment of each component (e.g., delay the visual response by $100 \mathrm{~ms}$ when the visual stimulus was presented $100 \mathrm{~ms}$ after the auditory one).

\section{Results}

\section{Visual stimuli elicited transient pupil dilation}

The sudden appearance of a visual stimulus resulted in a multiphasic pupil response (Fig. 2A). Transient pupil dilation was first elicited, followed by a constriction, and then second dilation, before pupil size returned to the control condition (no stimulus). For subsequent illustration and analysis, pupil responses evoked by stimuli (Fig. $2 A$, solid lines) were subtracted from the mean pupil response of the control condition (Fig. $2 A$, dotted line, $B$; see Materials and Methods).

Pupil size is sensitive to global changes in luminance (Loewenfeld, 1999), and a brief presentation of luminant stimuli could alter global luminance and therefore influence pupil size. If true, then the pupil responses induced by contrast increment- or decrementstimuli should be different. The presentation of positive contrast stimuli should result in a constriction because it increases overall illumination, whereas the presentation of negative contrast stimuli should cause initial dilation because it decreases overall illumination.
The results showed that initial transient pupil dilation was evoked regardless of stimulus luminance defined by positive or negative contrast (Pos/Neg) relative to background (Fig. 2B, brighter or dimmer solid lines, respectively), although statistically larger pupil dilation was elicited by the presentation of negative contrast stimuli. Moreover, a similar response was produced regardless of background luminance and across two different monkeys (Fig. $2 C-F$ ). Together, the results suggest that the evoked transient pupil response was mediated by a mechanism dissociable from the pupil light reflex pathway that regulates pupil size according to the global luminance (Loewenfeld, 1999; Gamlin, 2006). Given that the pupil responded similarly for each monkey regardless of background luminance and direction of visual contrast, these data were collapsed for subsequent analyses.

\section{Pupil response scaled with visual intensity}

Brief presentation of a visual stimulus produced transient pupil responses that were greater when high-contrast (more salient) stimuli were presented (Fig. 3A). The effects of stimulus contrast on the evoked pupil response were consistent across two monkeys (Fig. $3 A, B)$. Comparable effects on SRT were evident on saccade trials (Fig. 3C; high/low: monkey U, $150 \pm 3 \mathrm{~ms} / 158 \pm 5 \mathrm{~ms}$; monkey D, $176 \pm 4 \mathrm{~ms} / 188 \pm 3 \mathrm{~ms} ; \pm$ values were defined by $\pm 95 \%$ confidence interval and the comparison was statistically significant when two CIs did not overlap; see Materials and Methods), as shown previously (Marino and Munoz, 2009).

Visual stimulus contrast modulated the PROL, and the pupil responded faster for high-contrast stimuli (Fig. 3D; PROL: high- 


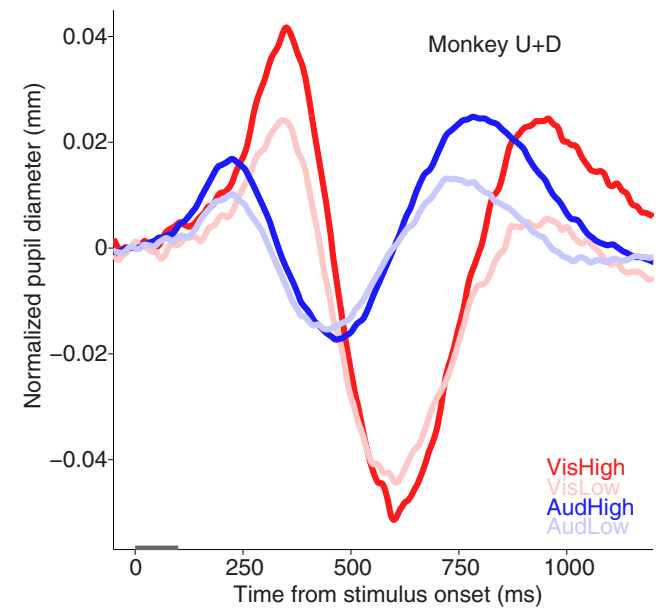

Figure 5. Comparison the evoked pupil response between visual and auditory modality. Similar pupil response evoked by presentation of visual or auditory stimulus. The red and blue colors indicate visual and auditory stimulus conditions, respectively. Gray bar on $x$-axis indicates the time line of stimulation. VisHigh, High salient visual stimulus; VisLow, low salient visual stimulus; AudHigh, high salient auditory stimulus; AudLow, low salient auditory stimulus.

\section{Auditory stimuli elicited transient pupil dilation}

If the above evoked pupil responses were driven by stimulus saliency, they should also be elicited with nonvisual stimuli. Figure $4 A$ illustrates the effects of auditory stimulus presentation on the pupil. Presentation of an auditory stimulus also resulted in a multiphasic pupil response that was similar to that elicited by a visual stimulus. The initial phasic response was evoked, with transient pupil dilation followed by a constriction, and secondary dilation before the pupil size merged with the no-stimulus condition. Two monkeys showed a similar pattern of auditory saliency modulation (Fig. $4 A, B$ ).

Manipulation of auditory intensity had similar effects on pupil size, although they did not reach significance. Auditory stimulus intensity influenced the timing of pupil responses: PROL was $159 \mathrm{~ms}$ for high-intensity auditory stimuli, and $223 \mathrm{~ms}$ for low-intensity stimuli (Fig. 4C; the observed pupil values on the low-intensity condition overlapped with the upper baseline pupil values, so we selected the time with the highest observed difference). Furthermore, presentation of a high-intensity auditory stimulus elicited a larger mean dilation (Fig. 4D; highintensity, $0.028 \pm 0.006 \mathrm{~mm}$; low-intensity, $0.021 \pm 0.007 \mathrm{~mm}$ ), peak dilation (Fig. $4 D$; high-intensity, $0.042 \pm 0.006 \mathrm{~mm}$; lowintensity, $0.035 \pm 0.007 \mathrm{~mm}$ ), and the peak-to-peak response (Fig. 4D; highintensity, $0.082 \pm 0.005 \mathrm{~mm}$; lowintensity, $0.075 \pm 0.005 \mathrm{~mm})$. However, there was no difference in the mean velocity of dilation (Fig. 4E; high-intensity, $0.17 \pm 0.05 \mathrm{~mm} / \mathrm{s}$; low-intensity, $0.17 \pm$ $0.06 \mathrm{~mm} / \mathrm{s}$ ).

\section{Similar transient pupil responses were} evoked by visual and auditory stimuli The presentation of a visual or auditory stimulus evoked a remarkably similar pattern of pupil responses (Fig. 5). Initial transient pupil dilation was evoked and followed by a constriction, and then secondary dilation appeared before pupil size merged with the control condition. A similar modulation of contrast-based saliency was evident for both visual and auditory modalities, with faster PROL (Figs. 3D, $4 B)$ and larger evoked pupil responses (Figs. 3E, 4D) for more salient stimuli, although the modulations with auditory stimuli did not reach significance. Importantly, the pupil responded earlier in time, but with smaller magnitude (Fig. 5) following the auditory stimuli. Note that we did not apply psychophysical methods to determine the pupil response threshold

contrast, $222 \mathrm{~ms}$; low-contrast, $259 \mathrm{~ms})$. Moreover, the sizerelated pupil responses were also influenced by stimulus contrast. High-contrast visual stimuli elicited statistically larger peak dilation (Fig. 3E; high contrast, $0.057 \pm 0.007 \mathrm{~mm}$; low-contrast, $0.041 \pm 0.008 \mathrm{~mm}$ ) and peak-to-peak response (Fig. 3E; high contrast, $0.13 \pm 0.007 \mathrm{~mm}$; low-contrast, $0.11 \pm 0.007 \mathrm{~mm}$ ), and the difference in mean dilation was marginally significant (Fig. $3 E$; high-contrast, $0.036 \pm 0.008 \mathrm{~mm}$; low-contrast, $0.025 \pm$ $0.008 \mathrm{~mm}$ ). The mean velocity of dilation was also modulated by visual contrast (Fig. 3F; high-contrast, $0.36 \pm 0.04 \mathrm{~mm} / \mathrm{s}$; lowcontrast, $0.23 \pm 0.07 \mathrm{~mm} / \mathrm{s})$. for visual or auditory stimuli, as we used suprathreshold stimuli to evoke pupil responses but made no attempt to match auditory and visual intensity. Thus, any observed differences in modality should be considered with caution.

\section{SCi-microstimulation evoked qualitatively similar transient pupil dilation}

These pupillary results were comparable to modulations in the SCi: increasing saliency of sensory stimuli evokes faster and larger visual responses in the SCi (Wise and Irvine, 1983; Marino et al., 2012), and auditory stimuli induce faster, but smaller sensory 
responses in the SCi than those produced with visual stimuli (Bell et al., 2004). Overall, the demonstration of similar pupil responses for auditory and visual stimuli further implicates the SCi in the circuit because of its central role in multisensory processing (Stein and Meredith, 1993) and coordinating the orienting response (Boehnke and Munoz, 2008).

If the observed sensory-evoked pupil response was part of the orienting response that mediated by the $\mathrm{SCi}$, then $\mathrm{SCi}$ microstimulation should also evoke pupil dilation. Microstimulation of the SCi subthreshold for saccades also produced a multiphasic pupil response (Fig. 6B; Wang et al., 2012). Although the pupil responses elicited by SCi microstimulation and visual stimulus presentation were different (Fig. 6, compare $A, B$ ), the general response pattern was similar: initial transient pupil dilation was followed by a constriction, and then a weak sustained dilation appeared before pupil size merged with the control condition. This transient pupil response evoked by $\mathrm{SCi}$ microstimulation was statistically significant on different background luminance conditions, although the size of pupil dilation was larger on the dark background condition. The initial transient pupil dilation was not correlated with a stimulation location on the SC map (Wang et al., 2012), so data from different SC sites were collapsed for further analyses. Because different levels of background illumination modulated the size of microstimulation-evoked pupil dilation (Wang et al., 2012), we only compared microstimulation results with visual stimulus task results because they had the same background luminance manipulations. Because visual and microstimulation experiments were performed by different monkeys, the pupil measurements were quantified individually (Fig. 6C-F).

The PROL was faster for SCi-microstimulation than visualstimulus evoked pupil responses by $\sim 60 \mathrm{~ms}$ (Fig. $6 C$; monkey Q and Y: 167 and $175 \mathrm{~ms}$, respectively; monkey $U$ and D: 220 and $242 \mathrm{~ms}$, respectively). These timing differences persisted when the pupil response reached its peak [SCi: $301 \pm 4 \mathrm{~ms}$ (monkey Q)/298 $\pm 4 \mathrm{~ms}$ (monkey Y); visual: $325 \pm 7 \mathrm{~ms}$ (monkey U)/ $346 \pm 9 \mathrm{~ms}$ (monkey D)]. This is consistent with the SC being in the pathway leading to saliency-induced changes in pupil size.

Although not equated for intensity, size-related measurements (mean and peak pupil dilation) were similar [Fig. $6 D$; mean dilation, SCi: $0.026 \pm 0.002 \mathrm{~mm}$ (monkey Q)/0.023 \pm $0.003 \mathrm{~mm}$ (monkey Y), visual: $0.033 \pm 0.007 \mathrm{~mm}$ (monkey $\mathrm{U}) / 0.021 \pm 0.006 \mathrm{~mm}$ (monkey D); Figure $6 E$; peak dilation, SCi: $0.049 \pm 0.003 \mathrm{~mm}$ (monkey Q) $/ 0.043 \pm 0.003 \mathrm{~mm}$ (monkey Y), visual: $0.047 \pm 0.007 \mathrm{~mm}($ monkey $\mathrm{U}) / 0.037 \pm 0.006$ $\mathrm{mm}$ (monkey D)], and on the mean dilation velocity [Fig. 6F; SCi: $0.032 \pm 0.002 \mathrm{~mm}$ (monkey Q)/0.031 $\pm 0.002 \mathrm{~mm}$ (mon- key Y), visual: $0.035 \pm 0.004 \mathrm{~mm}($ monkey $\mathrm{U}) / 0.027 \pm 0.005$ mm (monkey D)].

Moreover, the initial component of visually evoked response was similar to that of the microstimulation-evoked response, but delayed by the approximate difference in arrival of visual stimuli in the SCi. Although the SCi seems only to drive the initial increase of pupil dilation, the results are actually in line with the suggested role of the SCi in saccade generation, in that it may only drive the initial acceleration component of saccade profiles (Quaia et al., 1999). Together, the SCi could participate in the production of the transient pupil response, particularly the initial dilation, evoked by presentation of novel visual stimuli. Although the latency of microstimulation-evoked pupil dilation seems long relative to saccades, we used weak stimulation that was subthreshold for saccade initiation, and in general the pupil system acts more slowly than the saccade system, with constriction latencies $\sim 150 \mathrm{~ms}$ for the pupil light reflex to background luminance changes (Pong and Fuchs, 2000).

\section{Combination of visual and auditory pupil responses}

One study has suggested that the pupil responds linearly to a sequential presentation of visual and auditory stimuli (Hoeks and Levelt, 1993). Thus, we tested linear summation of pupil 


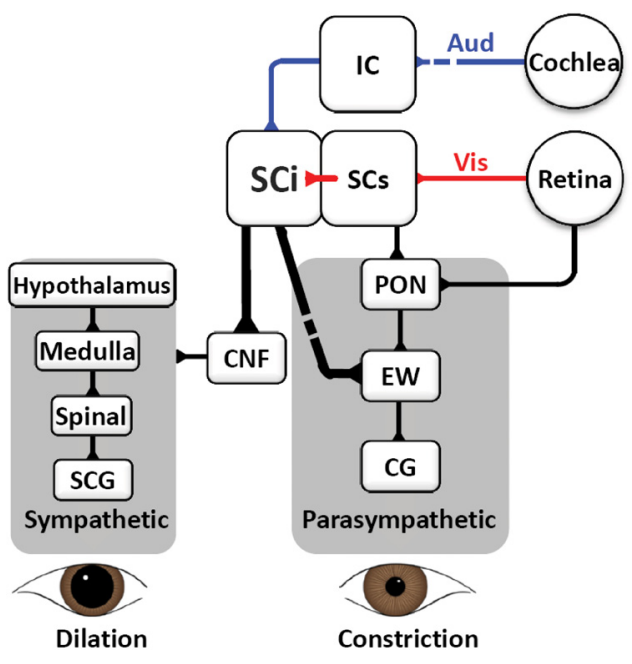

Figure 8. Schematic of the proposed neural pathway that underlies the salience-evoked pupil response. CG, Ciliary ganglion; CNF, mesencephalic cuneiform nucleus; EW, EdingerWestphal nucleus; IC, inferior colliculus, PON, pretectal olivary nucleus; $\mathrm{SCi}$, intermediate layers of superior colliculus; SCs, superficial layer of superior colliculus; SCG, superior cervical ganglion; Spinal: spinal cord.

responses elicited by the simultaneous or temporally offset presentation of visual and auditory stimuli. To examine the additive model, a separate experiment was conducted with monkey $\mathrm{U}$, in which visual and auditory stimuli were either presented alone or in combination. The presentation of a unimodal visual or auditory stimulus evoked the typical pupil response (Fig. 6A). The presentation of visual and auditory stimuli simultaneously induced similar pupil responses, and critically, the evoked pupil responses were comparable to those derived from summing the visual- and auditory-only pupil responses (Fig. 6B, Model-Add curve). We further tested the linear addition assumption by presenting two stimuli in sequence with $100 \mathrm{~ms}$ temporal asynchrony. The pupil responses between actual data and time-shifted addition of unimodal responses were similar regardless of the order of modality (Fig. 6C, Model-Add, auditory stimulus first; $D$, Model-Add, visual stimulus first). The simple additive model provided a good fit to the audiovisual data (root mean square errors: V0A, 0.29; A100V, 0.34; V100A, 0.28), although some qualitative features of the data were not predicted by the model. In conclusion, our modeling results suggest that the pupil, in general, responded in a linearly additive manner to audiovisual stimuli. Moreover, because the location of the auditory stimulus was not spatially aligned with the visual stimulus, it is possible that $\mathrm{SCi}$ activity induced by stimuli presented from different spatial locations could be integrated in a spatially nonlocalized manner to generate a coordinated pupil response.

\section{Discussion}

The orienting system responds to salient changes in the environment by initiating a coordinated orienting response that includes transient pupil dilation (Sokolov, 1963a; Lynn, 1966). In monkeys, the brief presentation of a visual or auditory stimulus evoked a reliable triphasic pupil response that included an initial dilation followed by a constriction and then a second dilation. The response was dissociated from illumination-dependent pupil modulation because it was evoked by a visual stimulus that was either a luminance increment or decrement relative to back- ground (Fig. 2B). Similar pupil responses were induced by auditory and visual stimuli (Fig. 5). Importantly, contrast-based saliency modulated the timing and magnitude of evoked pupil responses, with faster and larger pupil responses observed for higher contrast stimuli. Pupil responses to audiovisual stimuli could be accurately predicted by summing the component unisensory responses (Fig. 7). Our results suggest that the SCi are a likely substrate contributing to the transient pupil response because of its central role in multisensory processing (Stein and Meredith, 1993). Moreover, similar initial transient pupil dilation was evoked by both SCi microstimulation and presentation of salient stimuli, with onset latency differences that matched the arrival time of sensory signals in the SCi (Fig. 6). In summary, transient pupil responses, as one component of the orienting response, were modulated by contrast-based saliency, and the SCi likely coordinated the response.

\section{Pupil orienting response}

Pupil size varies continuously and this variation is largely attributed to global luminance changes (Loewenfeld, 1999; Gamlin, 2006). Pupil size is also modulated by salient events in the environment. It has long been suggested that the pupil response is related to orienting (Sokolov, 1963a; Lynn, 1966), and transient pupil dilation can be observed after the appearance of salient auditory stimuli (Stelmack and Siddle, 1982; Qiyuan et al., 1985; Netser et al., 2010; Steiner and Barry, 2011). Consistent with the literature, transient pupil dilation was consistently evoked in monkeys by presentation of either visual or auditory stimuli. Remarkably similar triphasic pupil responses were evoked by presenting visual or auditory stimuli, with faster but smaller responses following auditory stimuli. Stimulus contrast modulated not only the timing of the response, but also its magnitude, with faster and larger pupil responses following the presentation of higher contrast stimuli.

Although the auditory responses did not reach significance on some measurements, the lack of significance is likely due to the completely irrelevant nature of the auditory stimulus in the task; saccades to the visual stimulus, but not the auditory stimulus, were required on a subset of trials. Therefore, monkeys likely paid less attention to the auditory stimulus resulting in reduced responses (Hess and Polt, 1960). Moreover, it may have been difficult for the monkeys to make accurate spatially directed orienting responses because our study used a tonal stimulus and the monkey was head-fixed (Populin, 2006), which could also reduce the evoked pupil response by diminishing localizability. It is also possible that insufficient intensity differences were used for two auditory stimuli to reveal a difference. Nevertheless, a qualitatively similar saliency modulation was observed, with shorter latency and larger responses elicited by higher intensity auditory stimuli.

The current study manipulated contrast-based saliency because it is one of most primitive saliency components (Itti and Koch, 2001) and is widely used for computational modeling (Borji et al., 2013). However, the saliency computation defines how conspicuous the stimulus is relative to the background, thus involving the competition of all available stimuli. For example, a red stimulus would be more salient among a group of green stimuli than a group of red stimuli. Our results only demonstrate modulation of pupil size by contrast-based saliency, and future research is required to address modulation by other aspects of saliency. 


\section{Possible functions of the pupil orienting response}

The orienting response facilitates allocation of neuronal resources toward potentially important objects or locations to prepare the body for appropriate actions. It has been suggested that transient pupil dilation can slightly increase visual sensitivity (Sokolov, 1963a; Stelmack and Siddle, 1982), although there is no direct evidence to support this. Importantly, it is not clear how the different orienting components are coordinated to optimize performance. Our results showed that the PROL for visual stimuli was $\sim 225 \mathrm{~ms}$, which is slightly longer than the SRTs elicited by similar salient visual stimuli, $\sim 150-$ $200 \mathrm{~ms}$ (Marino and Munoz, 2009). Furthermore, the time required for a spatial attention shift induced by a peripheral cue (stimulus-driven) was regularly within $100 \mathrm{~ms}$ (Carrasco, 2011). It is thus possible that the appearance of a salient visual stimulus first leads to a shift of attention and gaze to the object to enhance its recognition. Immediately after foveation, the pupil starts to dilate, presumably to slightly increase visual sensitivity of the targeted object and its surrounding locations, providing a possible benefit for pupil dilation latency to be longer than saccade latency. This coordination can facilitate processing of the selected object to optimize an organism's performance.

\section{The role of the SC in the pupil orienting response}

The SC is organized into a retinotopic map of contralateral visual space with functionally and anatomically differentiated layers. The superficial layers receive inputs from early visual areas exclusively, including the retina and the primary visual cortex, whereas the SCi receives inputs from the superficial SC as well as multisensory and frontal-parietal areas, and then projects directly to the brainstem and the spinal cord to execute the orienting response (White and Munoz, 2011). Because the SCi integrates sensory-related and goal-directed signals from cortical and subcortical areas, it is hypothesized to encode stimuli based upon saliency and relevance to coordinate the orienting response (Sparks, 1986; Fecteau and $\mathrm{Mu}-$ noz, 2006; Knudsen, 2007; Boehnke and Munoz, 2008; Mysore and Knudsen, 2013). Microstimulation of the SCi not only induces saccades (Robinson, 1972) and biases spatial attention (Kustov and Robinson, 1996; Cavanaugh and Wurtz, 2004; Müller et al., 2005), but also evokes transient pupil responses (Netser et al., 2010; Wang et al., 2012). The similar pupil responses evoked by presentation of both salient visual and auditory stimuli and the qualitatively similar transient pupil dilation elicited by SCi microstimulation suggests that the SCi is likely involved in control of salience-evoked pupil responses.

Consistent with our observations on evoked pupil responses, changing the level of stimulus contrast modulates the timing and magnitude of sensory responses in the SCi (Wise and Irvine, 1983; Marino et al., 2012), with more salient stimuli inducing faster and larger SC sensory responses. Sensory responses in the SCi are also faster but smaller following the presentation of auditory compared with visual stimuli (Bell et al., 2004), consistent with our observations of the effect of modality on pupil modulation. Given these consistencies, we propose that the sensory responses in the SCi induced by different modalities and different levels of stimulus contrast systematically influence the timing and magnitude of orienting pupil responses.

\section{Possible anatomical pathways underlying the salience-related pupil response}

Pupil size is controlled by the interactions between the parasympathetic and sympathetic pathways (Loewenfeld, 1999). The parasympathetic pathway underlies the illumination-dependent pupil modulation to optimize visual acuity (Leibowitz, 1952; Campbell and Gregory, 1960; Woodhouse, 1975; Laughlin, 1992). Information about illumination is carried directly from the retina to the pretectal olivary nucleus (PON). PON neurons project bilaterally to the Edinger-Westphal (EW) nucleus, which contains the parasympathetic, preganglionic neurons that project to the ciliary ganglion to control pupillary constriction muscles of the iris (Loewenfeld, 1999; Gamlin, 2006). In addition, pupil size is also controlled by the dilator muscle, innervated by sympathetic nerves from the superior cervical ganglion, which is driven by a sympathetic circuit originating in the hypothalamus (Loewenfeld, 1999).

Because weak microstimulation of the superficial SC did not evoke pupil dilation (Wang et al., 2012), pupil dilation should be mediated via connections from the SCi to the pupil pathways (Fig. 8). The SCi could inhibit parasympathetic pathways via indirect inhibitory projections to the EW (Edwards and Henkel, 1978; Harting et al., 1980; Grantyn and Grantyn, 1982) or can activate parasympathetic pathways via direct excitatory projections to the EW (Harting et al., 1980). The SCi also projects to the mesencephalic cuneiform nucleus (CNF) (Harting, 1977; Huerta and Harting, 1984; May, 2006), a brainstem area regulating stress-related and defensive responses (Dean et al., 1989; Korte et al., 1992). Stimulation of the CNF activates sympathetic vasomotor outflow (Verberne, 1995), which also influences pupil dilation (Loewenfeld, 1999). We propose that projections from the SCi to the EW and the CNF may underlie this response either by inhibiting the parasympathetic (pupil constriction) pathway, activating the sympathetic (pupil dilation) pathway, or both. The SC contrast-based saliency coding could be translated to the pupil through the suggested pathways.

\section{References}

Bala AD, Takahashi TT (2000) Pupillary dilation response as an indicator of auditory discrimination in the barn owl. J Comp Physiol A 186:425-434. CrossRef Medline

Beatty J (1982) Task-evoked pupillary responses, processing load, and the structure of processing resources. Psychol Bull 91:276-292. CrossRef Medline

Beatty J, Lucero-Wagoner B (2000) The pupillary system. In: The handbook of psychophysiology (Caccioppo J, Tassinary LG, Berntson G, eds). Cambridge: UP.

Bell AH, Fecteau JH, Munoz DP (2004) Using auditory and visual stimuli to investigate the behavioral and neuronal consequences of reflexive covert orienting. J Neurophysiol 91:2172-2184. CrossRef Medline

Berg DJ, Boehnke SE, Marino RA, Munoz DP, Itti L (2009) Free viewing of dynamic stimuli by humans and monkeys. J Vis 9(5):19 1-15. CrossRef Medline

Boehnke SE, Munoz DP (2008) On the importance of the transient visual response in the superior colliculus. Curr Opin Neurobiol 18:544-551. CrossRef Medline

Borji A, Sihite DN, Itti L (2013) Quantitative analysis of human-model agreement in visual saliency modeling: a comparative study. IEEE Trans Image Process 22:55-69. CrossRef Medline

Campbell FW, Gregory AH (1960) Effect of size of pupil on visual acuity. Nature 187:1121-1123. CrossRef Medline

Carrasco M (2011) Visual attention: the past 25 years. Vision Res 51:14841525. CrossRef Medline

Cavanaugh J, Wurtz RH (2004) Subcortical modulation of attention counters change blindness. J Neurosci 24:11236-11243. CrossRef Medline

Dean P, Redgrave P, Westby GW (1989) Event or emergency? two response systems in the mammalian superior colliculus. Trends Neurosci 12:137147. CrossRef Medline 
Desimone R, Duncan J (1995) Neural mechanisms of selective visual attention. Annu Rev Neurosci 18:193-222. CrossRef Medline

Edwards SB, Henkel CK (1978) Superior colliculus connections with the extraocular motor nuclei in the cat. J Comp Neurol 179:451-467. CrossRef Medline

Eldar E, Cohen JD, Niv Y (2013) The effects of neural gain on attention and learning. Nat Neurosci 16:1146-1153. CrossRef Medline

Fecteau JH, Munoz DP (2006) Salience, relevance, and firing: a priority map for target selection. Trends Cogn Sci 10:382-390. CrossRef Medline

Gamlin PD (2006) The pretectum: connections and oculomotor-related roles. Prog Brain Res 151:379-405. CrossRef Medline

Grantyn A, Grantyn R (1982) Axonal patterns and sites of termination of cat superior colliculus neurons projecting in the tecto-bulbo-spinal tract. Exp Brain Res 46:243-256. CrossRef Medline

Hall WC, Moschovakis A (2004) he superior colliculus: new approaches for studying sensorimotor integration. Boca Raton, FL: CRC.

Harting JK (1977) Descending pathways from the superior collicullus: an autoradiographic analysis in the rhesus monkey (Macaca mulatta). J Comp Neurol 173:583-612. CrossRef Medline

Harting JK, Huerta MF, Frankfurter AJ, Strominger NL, Royce GJ (1980) Ascending pathways from the monkey superior colliculus: an autoradiographic analysis. J Comp Neurol 192:853-882. CrossRef Medline

Hays AV, Richmond BJ, Optican LM (1982) A UNIX-based multiple process system for real-time data acquisition and control. WESCON Conf Proc 2:1-10.

Hess EH, Polt JM (1960) Pupil size as related to interest value of visual stimuli. Science 132:349-350. CrossRef Medline

Hoeks B, Levelt WJ (1993) Pupillary dilation as a measure of attention: a quantitative system analysis. Behav Res Meth Instr Comput 25:16-26. CrossRef

Huerta M, Harting J (1984) Connectional organization of the superior colliculus. Trends Neurosci 7:286-289. CrossRef

Ingle D (1973) Evolutionary perspectives on the function of the optic tectum. Brain Behav Evol 8:211-237. CrossRef Medline

Itti L, Koch C (2000) A saliency-based search mechanism for overt and covert shifts of visual attention. Vision Res 40:1489-1506. CrossRef Medline

Itti L, Koch C (2001) Computational modelling of visual attention. Nat Rev Neurosci 2:194-203. CrossRef Medline

Knudsen EI (2007) Fundamental components of attention. Annu Rev Neurosci 30:57-78. CrossRef Medline

Koch C, Ullman S (1985) Shifts in selective visual attention: towards the underlying neural circuitry. Hum Neurobiol 4:219-227. Medline

Korte SM, Jaarsma D, Luiten PG, Bohus B (1992) Mesencephalic cuneiform nucleus and its ascending and descending projections serve stress-related cardiovascular responses in the rat. J Auton Nerv Syst 41:157-176. CrossRef Medline

Kowler E (2011) Eye movements: the past 25 years. Vision Res 51:14571483. CrossRef Medline

Krauzlis RJ, Lovejoy LP, Zénon A (2013) Superior colliculus and visual spatial attention. Annu Rev Neurosci 36:165-182. CrossRef Medline

Kustov AA, Robinson DL (1996) Shared neural control of attentional shifts and eye movements. Nature 384:74-77. CrossRef Medline

Laughlin SB (1992) Retinal information capacity and the function of the pupil. Ophthalmic Physiol Opt 12:161-164. Medline

Leibowitz H (1952) The effect of pupil size on visual acuity for photometrically equated test fields at various levels of luminance. J Opt Soc Am 42:416-422. CrossRef Medline

Loewenfeld IE (1999) The pupil: anatomy, physiology, and clinical applications. Boston: Butterworth-Heinemann.

Lynn R (1966) Attention, arousal and the orientation reaction. Oxford, UK: Pergamon.

Marino RA, Munoz DP (2009) The effects of bottom-up target luminance and top-down spatial target predictability on saccadic reaction times. Exp Brain Res 197:321-335. CrossRef Medline

Marino RA, Rodgers CK, Levy R, Munoz DP (2008) Spatial relationships of visuomotor transformations in the superior colliculus map. J Neurophysiol 100:2564-2576. CrossRef Medline

Marino RA, Levy R, Boehnke S, White BJ, Itti L, Munoz DP (2012) Linking visual response properties in the superior colliculus to saccade behavior. Eur J Neurosci 35:1738-1752. CrossRef Medline

May PJ (2006) The mammalian superior colliculus: laminar structure and connections. Prog Brain Res 151:321-378. CrossRef Medline
McPeek RM, Keller EL (2004) Deficits in saccade target selection after inactivation of superior colliculus. Nat Neurosci 7:757-763. CrossRef Medline

Middlebrooks JC, Green DM (1991) Sound localization by human listeners. Annu Rev Psychol 42:135-159. CrossRef Medline

Moresi S, Adam JJ, Rijcken J, Van Gerven PW (2008) Cue validity effects in response preparation: a pupillometric study. Brain Res 1196:94-102. CrossRef Medline

Müller JR, Philiastides MG, Newsome WT (2005) Microstimulation of the superior colliculus focuses attention without moving the eyes. Proc Natl Acad Sci U S A 102:524-529. CrossRef Medline

Mysore SP, Knudsen EI (2013) A shared inhibitory circuit for both exogenous and endogenous control of stimulus selection. Nat Neurosci 16:473478. CrossRef Medline

Nassar MR, Rumsey KM, Wilson RC, Parikh K, Heasly B, Gold JI (2012) Rational regulation of learning dynamics by pupil-linked arousal systems. Nat Neurosci 15:1040-1046. CrossRef Medline

Netser S, Ohayon S, Gutfreund Y (2010) Multiple manifestations of microstimulation in the optic tectum: eye movements, pupil dilations, and sensory priming. J Neurophysiol 104:108-118. CrossRef Medline

Parkhurst D, Law K, Niebur E (2002) Modeling the role of salience in the allocation of overt visual attention. Vision Res 42:107-123. CrossRef Medline

Pong M, Fuchs AF (2000) Characteristics of the pupillary light reflex in the macaque monkey: discharge patterns of pretectal neurons. J Neurophysiol 84:964-974. Medline

Populin LC (2006) Monkey sound localization: head-restrained versus head-unrestrained orienting. J Neurosci 26:9820-9832. CrossRef Medline

Qiyuan J, Richer F, Wagoner BL, Beatty J (1985) The pupil and stimulus probability. Psychophysiology 22:530-534. CrossRef Medline

Quaia C, Lefevre P, Optican LM (1999) Model of the control of saccades by superior colliculus and cerebellum. J Neurophysiol 82:999-1018. Medline

Robinson DA (1963) A method of measuring eye movement using a scleral search coil in a magnetic field. IEEE Trans Biomed Eng 10:137-145. Medline

Robinson DA (1972) Eye movements evoked by collicular stimulation in the alert monkey. Vision Res 12:1795-1808. CrossRef Medline

Schiller PH, Sandell JH, Maunsell JH (1987) The effect of frontal eye field and superior colliculus lesions on saccadic latencies in the rhesus monkey. J Neurophysiol 57:1033-1049. Medline

Sokolov EN (1963a) Perception and the conditioned reflex. Oxford: Pergamon.

Sokolov EN (1963b) Higher nervous functions; the orienting reflex. Annu Rev Physiol 25:545-580. CrossRef Medline

Sparks DL (1986) Translation of sensory signals into commands for control of saccadic eye movements: role of primate superior colliculus. Physiol Rev 66:118-171. Medline

Stein B, Meredith M (1993) The merging of the senses. Cambridge, MA: MIT.

Steiner GZ, Barry RJ (2011) Pupillary responses and event-related potentials as indices of the orienting reflex. Psychophysiology 48:1648-1655. CrossRef Medline

Stelmack RM, Siddle DA (1982) Pupillary dilation as an index of the orienting reflex. Psychophysiology 19:706-708. CrossRef Medline

Verberne AJ (1995) Cuneiform nucleus stimulation produces activation of medullary sympathoexcitatory neurons in rats. Am J Physiol 268:R752R758. Medline

Wang CA, Boehnke SE, White BJ, Munoz DP (2012) Microstimulation of the monkey superior colliculus induces pupil dilation without evoking saccades. J Neurosci 32:3629-3636. CrossRef Medline

White BJ, Munoz DP (2011) The superior colliculus. In: Oxford handbook of eye movements (Liversedge S, Gilchrist I, Everling S, eds). Oxford: Oxford UP.

Wierda SM, van Rijn H, Taatgen NA, Martens S (2012) Pupil dilation deconvolution reveals the dynamics of attention at high temporal resolution. Proc Natl Acad Sci U S A 109:8456-8460. CrossRef Medline

Wise LZ, Irvine DR (1983) Auditory response properties of neurons in deep layers of cat superior colliculus. J Neurophysiol 49:674-685. Medline

Woodhouse JM (1975) The effect of pupil size on grating detection at various contrast levels. Vision Res 15:645-648. CrossRef Medline 